\title{
A Novel Symmetrical Heuristic Coefficient for Urban Microcellular Environments
}

\author{
Puspraj Singh Chauhan, Member, IACSIT and Sanjay Soni
}

\begin{abstract}
A novel heuristic diffraction coefficient is presented which is perfectly reciprocal and symmetrical. The prediction obtained using proposed coefficient is compared with that obtained using rigorous Maliuzhinets' solution. It is shown that the proposed coefficient is more efficient than available diffraction coefficient. The comparison is made for both soft and hard polarization. Further, the applicability of proposed coefficient in complex urban scenario is demonstrated by applying the coefficient in the city of Ottawa.
\end{abstract}

Index Terms-Deterministic propagation model, microcellular scenario, ray tracing, uniform theory of diffraction (UTD).

\section{INTRODUCTION}

Geometrical theory of diffraction (GTD) [1] and Uniform theory of diffraction (UTD) [2] are high frequency asymptotic solution to the problem of diffraction by a wedge. The modeling of wireless propagation channel based on deterministic approach is usually performed using Geometrical theory of diffraction (GTD) and its extension uniform theory of diffraction (UTD) [1], [2]. GTD gives fair prediction of the diffracted field at the points away from the shadow boundaries but fails to predict the field at the shadow boundaries. Uniform theory of diffraction which is an extension of GTD is based on Clemmow method of steepest descent gives continuous field at the boundaries (though not accurate). In order to make the UTD applicable for lossy dielectric wedge, this is modified by Luebbers [3] by heuristically incorporating Fresnel reflection coefficient as a multiplying factor to the components of diffraction coefficient. As a result, the coefficient becomes applicable for dielectric wedge. However, it lacks accuracy in certain region such as shadow region (e.g. See [4], Fig.13, 14). Holm [5] proposed modification of the original Luebbers coefficient by modifying the multiplying factors to be used in the coefficient. This resulted in the improvement in the accuracy of shadow region. However, Holm's coefficient lacked accuracy in the illumination region. The modification to Luebbers formulation was proposed by Kate A. Remley et al. [4] who modified the angles to be used in the calculation of Reflection coefficient. As a result, this improved the accuracy in the shadow region. Modification to the Holm's

Manuscript received February 15, 2012; revised March 13, 2012

P. Singh Chauhan is with the Department of Electronics and Telecommunication College of Engineering Roorkee, Roorkee, India (e-mail: Puspraj.chauhan@gmail.com).

S. Soni is with the Department of Electronics and Telecommunication, G. B. Pant Engineering College, Pauri, India (e-mail: sanjoo_ksoni@yahoo.co.uk). coefficient was proposed by El-Sallabi et al. [6]. In this, for exterior and interior wedge both, the angle definition used in Fresnel reflection coefficient in the calculation of diffraction coefficient were defined extensively. They show good agreement with the rigorous Maliuzhinets solution. However, these coefficients were neither reciprocal nor symmetrical. In [7], a reciprocal heuristic coefficient was defined that used the angle definition proposed by Aidi et al. [9] and showed good agreement over other available coefficients. However, this showed the reciprocity property only when the transmitter (Tx) and the receiver (Rx) were either side of the wedge. When the Tx and Rx were both on the same side, it was not reciprocal. Moreover, it does not show symmetry property.

The present work proposes the heuristic diffraction coefficient which is perfectly reciprocal and symmetrical. Moreover, it is shown that the coefficient is more efficient over the other available diffraction coefficients. In order to validate the coefficient, the comparison is done with rigorous Maliuzhinets coefficient and available measurement.

The paper is organized as follows. The section-II gives the problem formulation and brief description of available heuristic coefficient. Section-III deals with the proposed solution and detailed discussion of the reciprocal and symmetrical property of diffraction coefficient. Section-IV deals with the results and discussion followed by conclusion.

\section{AvaILABLE HeURISTIC DifFraction COEFFICIENT}

The problem of diffraction from the dielectric wedge can be observed in Fig. 1. The source is at the distance $r_{1}$ from the wedge tip and the observation point is at the distance $r_{2}$ from the tip. The angle to the incident ray and the diffracted ray from the 0 -face are $\phi$ and $\phi^{\prime}$ respectively. The diffracted field at the observation point is given as

$$
E(r)=\frac{E_{0}}{r_{1}} D \sqrt{\frac{r_{1}}{r_{2}\left(r_{1}+r_{2}\right)}} \exp \left(-j k\left(r_{1}+r_{2}\right)\right)
$$

where $E_{0}$ the transmitted field at the transmitter and D is the diffraction coefficient of the wedge. For perfect electrical conducting wedge (PEC), the diffraction coefficient proposed by Pathak et al. [2] is given by

$$
D_{S, h}=-\frac{e^{-j \frac{\pi}{4}}}{2 n \sqrt{2 \pi k}}\left(D_{1}+D_{2} \mp\left(D_{3}+D_{4}\right)\right)
$$




$$
\begin{aligned}
& D_{i}=\cot \beta_{i} \times F\left[k L X_{i}\right], i=1, \ldots \ldots 4 \\
& \beta_{1}=\frac{\pi+\left(\phi-\phi^{\prime}\right)}{2 n}, X_{1}=a^{+}\left(\phi-\phi^{\prime}\right) \\
& \beta_{2}=\frac{\pi-\left(\phi-\phi^{\prime}\right)}{2 n}, X_{2}=a^{-}\left(\phi-\phi^{\prime}\right) \\
& \beta_{3}=\frac{\pi+\left(\phi+\phi^{\prime}\right)}{2 n}, X_{3}=a^{+}\left(\phi+\phi^{\prime}\right) \\
& \beta_{4}=\frac{\pi-\left(\phi+\phi^{\prime}\right)}{2 n}, X_{4}=a^{-}\left(\phi+\phi^{\prime}\right)
\end{aligned}
$$

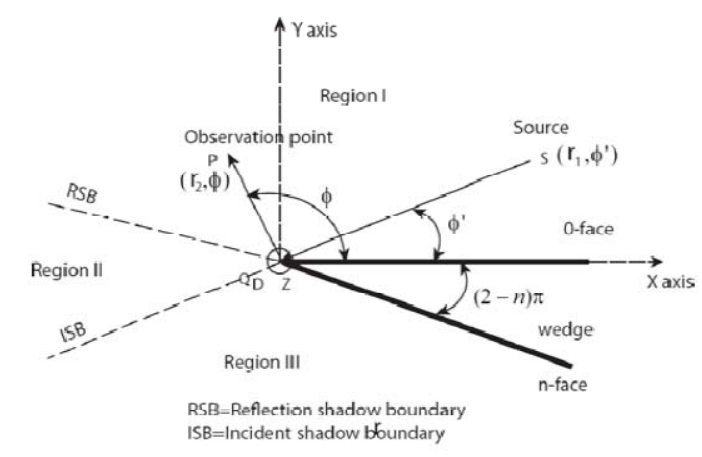

Fig. 1. An illustration of diffraction by dielectric wedge.

For lossy dielectric wedge, Luebbers modified (2) by using multiplying factors as follows

$$
D_{S, h}=-\frac{e^{-j \frac{\pi}{4}}}{2 n \sqrt{2 \pi k}}\left(D_{1}+D_{2}+R_{n}^{s, h} D_{3}+R_{0}^{s, h} D_{4}\right)
$$

where $R_{0}^{s, h}$ and $R_{n}^{s, h}$ are the Fresnel reflectionCoefficient for 0 -face and $\mathrm{n}$-face respectively. Superscripts $\mathrm{s}$ and $\mathrm{h}$ stand for soft and hard polarization respectively

$$
\begin{aligned}
& R^{s}=\frac{\sin \theta-\sqrt{\varepsilon-\cos ^{2} \theta}}{\sin \theta+\sqrt{\varepsilon-\cos ^{2} \theta}} \\
& R^{h}=\frac{\varepsilon \sin \theta-\sqrt{\varepsilon-\cos ^{2} \theta}}{\varepsilon \sin \theta+\sqrt{\varepsilon-\cos ^{2} \theta}}
\end{aligned}
$$

Here, $\theta_{0}=\min \left(\phi^{\prime}, \phi\right)$, and $\theta_{n}=\min \left(n \pi-\phi^{\prime}, n \pi-\right.$ $\phi)$. Holm [5] rearranged the multiplying factors to obtain better performance than Luebber's diffraction coefficient in the deep shadow region. The Holm's heuristic diffraction coefficient is

given as

$$
D_{S, h}=-\frac{e^{-j \frac{\pi}{4}}}{2 n \sqrt{2 \pi k}}\left(R_{n}^{s, h} R_{0}^{s, h} D_{1}+D_{2}+R_{n}^{s, h} D_{3}+R_{0}^{s, h} D_{4}\right)
$$

Here, $R_{0}, R_{n}$ are calculated based on (9),(10). Daniela et al. [7] modified the work of Holm's diffraction coefficient by making it reciprocal. The Daniela's coefficient for soft and hard polarizations are given by

$$
D_{S, h}=-\frac{e^{-j \frac{\pi}{4}}}{2 n \sqrt{2 \pi k}}\left(M_{n}^{s, h} D_{1}+M_{0}^{s, h} D_{2}+R_{n}^{s, h} D_{3}+R_{0}^{s, h} D_{4}\right)
$$

where

$$
\begin{gathered}
M_{n}^{s, h}= \begin{cases}R_{n}^{s, h} R_{0}^{s, h} & \emptyset^{\prime} \leq \frac{\mathrm{n} \pi}{2} \\
1 & \emptyset^{\prime} \geq \frac{\mathrm{n} \pi}{2}\end{cases} \\
M_{0}^{s, h}=\left\{\begin{array}{cc}
1 & \emptyset^{\prime} \leq \frac{\mathrm{n} \pi}{2} \\
R_{n}^{s, h} R_{0}^{s, h} & \emptyset^{\prime} \geq \frac{\mathrm{n} \pi}{2}
\end{array}\right.
\end{gathered}
$$

Here, Fresnel reflection coefficient, $R_{0}, R_{n}$ are calculated with angle $\theta_{0}$ and $\theta_{n}$ as per angular definition of Aidi and Lavergnat [9] given as

$$
\theta_{0}=\theta_{n}=\min \left(\phi^{\prime}, \phi, n \pi-\phi^{\prime}, n \pi-\phi\right)
$$

It may be noted that the above diffraction coefficient is reciprocal when Tx and Rx are opposite side of the wedge

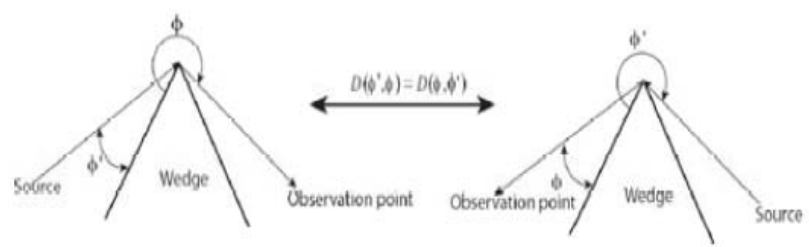

Fig. 2. Reciprocal condition of diffraction coefficient.

\section{RECIPROCAL AND SYMMETRICAL PROPERTY OF DIFFRACTION COEFFICIENT}

Diffraction coefficient is reciprocal if exchanging the position of the transmitter and the receiver does not alter the value of the diffraction coefficient. The condition is shown in Fig. (2). Hence,

$$
D\left(\phi, \phi^{\prime}\right)=D\left(\phi^{\prime}, \phi\right)
$$

Considering the formulation of diffraction coefficient in (2) to (7), we note that exchanging $\phi$ by $\phi^{\prime}$ results in $D_{1} \leftrightarrow D_{2}$ and $D_{3}, D_{4}$ remain unaltered. Therefore, our multiplying factors of $D_{1}, D_{2}$ should get interchanged with $\phi \leftrightarrow \phi^{\prime}$ and multiplying factors of $D_{3}, D_{4}$ should remain unchanged to make diffraction coefficient perfectly reciprocal.

Similarly, diffraction coefficient is said to be symmetrical if the value of the diffraction coefficient remains same irrespective of the 0 -face or $n$-face is taken as the reference face for angle measurement (See Fig. 3). Hence

$$
D\left(\phi, \phi^{\prime}\right)=D\left(n \pi-\phi, n \pi-\phi^{\prime}\right)
$$

In the expression of (2), it may be noted that with $\phi \leftrightarrow$ $n \pi-\phi$ and $\phi^{\prime} \leftrightarrow n \pi-\phi^{\prime}$ results in $D_{1} \leftrightarrow D_{2}$ and $D_{3} \leftrightarrow$ $D_{4}$ As results, the multiplying factors of these components pair should get interchanged to ensure symmetry property of the diffraction coefficient 


\section{Proposed Diffraction COEFFICIENT}

In the proposed diffraction coefficient, we divide the exterior angle of the dielectric wedge in the three region based on the position of the reflection shadow boundary (RSB) for 0 -face and $n$-face. Our proposed diffraction coefficient is as follows:

$$
D_{S, h}=-\frac{e^{-j \frac{\pi}{4}}}{2 n \sqrt{2 \pi}}\left(M_{1} D_{1}+M_{2} D_{2}+M_{3} D_{3}+M_{4} D_{4}\right)
$$

For reciprocal condition $M_{1 \leftrightarrow} M_{2}$ and $M_{3}=M_{4}$. For symmetrical property to be satisfied, we need $M_{1 \leftrightarrow} M_{2}$ and $M_{3} \leftrightarrow M_{4}$ these two conditions can be satisfied simultaneously when $M_{1 \leftrightarrow} M_{2}$ and $M_{3}=M_{4}$. Thus, we set multiplying factors $M_{i}, \mathrm{i}=1, \ldots, 4$ as described as in Table-I

In addition to that, for region-I,

$$
\theta_{0}=\theta_{n}=\min \left(T_{1}, T_{2}\right)
$$

Where $T_{1}=\frac{\pi}{2}-\left|\frac{\pi}{2}, \phi^{\prime}\right|$ and $T_{2}=\frac{\pi}{2}-\left|\frac{\pi}{2}, \phi\right|$ in Region-II,

$$
\theta_{0}=\theta_{n}=\min \left(\phi^{\prime}, \phi, n \pi-\phi^{\prime}, n \pi-\phi\right)
$$

TABLE I: MultiPlying FACTOR TO THE PROPOSED DifFRACTION COEFFICIENT

\begin{tabular}{|l|l|}
\hline \multicolumn{2}{|c|}{ Region-I,II,III } \\
\hline$\phi>\phi^{\prime}$ & $\phi<\phi^{\prime}$ \\
\hline$M_{1}=R_{0}\left(\theta_{0}\right) R_{n}\left(\theta_{n}\right)$ & $M_{1}=1$ \\
\hline$M_{2}=1$ & $M_{2}=R_{0}\left(\theta_{0}\right) R_{n}\left(\theta_{n}\right)$ \\
\hline$M_{3}=R_{0}\left(\theta_{0}\right)$ & $M_{3}=R_{0}\left(\theta_{0}\right)$ \\
\hline$M_{4}=R_{n}\left(\theta_{n}\right)$ & $M_{4}=R_{n}\left(\theta_{n}\right)$ \\
\hline
\end{tabular}

in Region-III,

$$
\begin{gathered}
\theta_{0}=\theta_{n}=\min \left(T_{3}, T_{4}\right) \\
T_{4}=\frac{\pi}{2}-\left|\frac{\pi}{2},(n \pi-\phi)\right| \\
T_{3}=\frac{\pi}{2}-\left|\frac{\pi}{2},\left(n \pi-\phi^{\prime}\right)\right|
\end{gathered}
$$

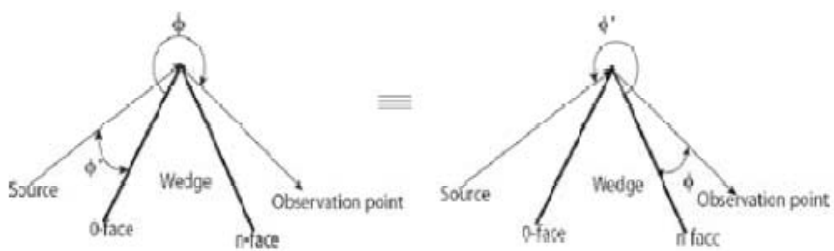

Fig. 3. Symmetrical condition of diffraction coefficient

\section{RESUlt AND Discussion}

\section{A. Symmetrical and Reciprocal Property Verification of} the Diffraction Coefficient

In this section, we consider the symmetrical property of the proposed coefficient. For this case, we consider a dielectric right-angle wedge. The incident angle of the ray that illuminates the tip of wedge is $135^{\circ}$ and the receiver moves in the circle of $2 \mathrm{~m}$ with an increment of $1^{0}$. In the first case, the angle measurement is done with respect to 0 -face and in the second case; it is measured with respect to the n-face. The result is shown in Fig. 4. Similarly, for reciprocal property, the incident angle is chosen to be and in the first case, incident angle is held constant to be $45^{\circ}$ and observation point is moved in a circle of the radius $2 \mathrm{~m}$. The sample interval is chosen to be $2^{0}$. In both the cases, hard polarization has been considered. The result is shown in Fig. 5.

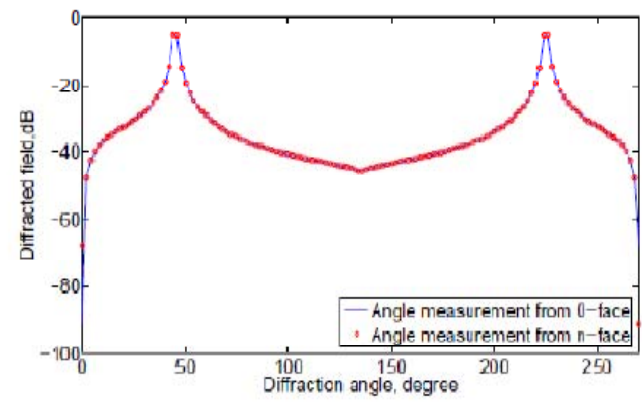

Fig. 4. Comparison of diffraction field obtained from the angle measurement from 0 -face and $n$-face.

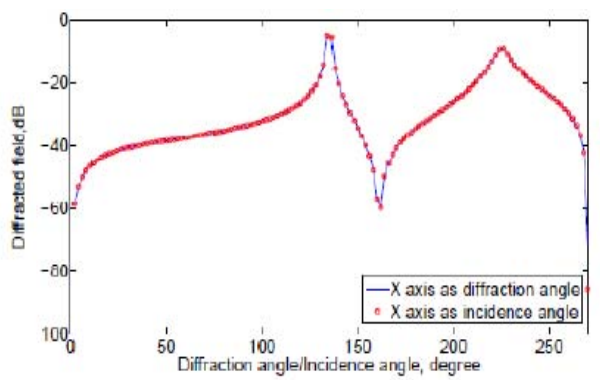

Fig. 5. Comparison of diffraction fields obtained from interchanging the

$$
\text { position of } T_{X} \text { and } R_{X} \text {. }
$$

\section{B. Comparison of Diffraction Coefficient with Rigorous} Maulizhinets Coefficients.

In this section, our objective is to show the usefulness of the proposed diffraction coefficient. The comparison of proposed diffraction coefficient is done with rigorous Maliuzhinets diffraction coefficient [8] and Holm's diffraction coefficient [5]. The wedge is characterized with 
conductivity $\sigma=0: 005 \mathrm{~S} / \mathrm{m}$ and permittivity $\mathcal{E}_{r}=6$. The distance of source and observation point was taken to be $3 \mathrm{~m}$ each from the tip of wedge. Keeping the source at given angle, the observation point was moved at the step of $2^{0}$ to obtain diffracted field. For comparison purpose, the wedge angle is chosen to be $10^{\circ}$ and $90^{\circ}$. Both the parallel and perpendicular polarizations are considered. Fig. 6 and Fig. 7 show the diffracted field pattern for the $10^{\circ}$ wedge and incident angle $45^{\circ}$ with both parallel and perpendicular polarizations. Here, a clear improvement can be seen in illumination region where Holm is differing from Maliuzhinets coefficient. Fig. 8 and Fig. 9 are for the $90^{0}$ wedge and incidence angle $45^{\circ}$. In this scenario, the novel coefficient gives good agreement with rigorous Maliuzhinets' solution viz-a-viz Holm's coefficient.

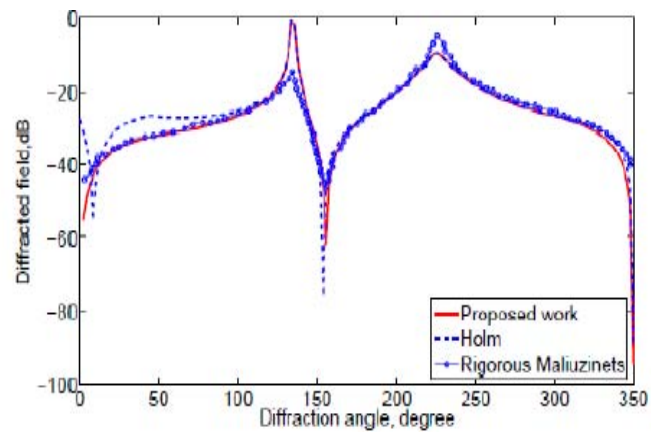

Fig. 6. Comparison of proposed diffraction coefficient with available diffraction coefficient and Rigorous Maulizhinets' solution [8]: Incident angle is $45^{\circ}$ and wedge angle is $10^{\circ}$, parallel polarization.

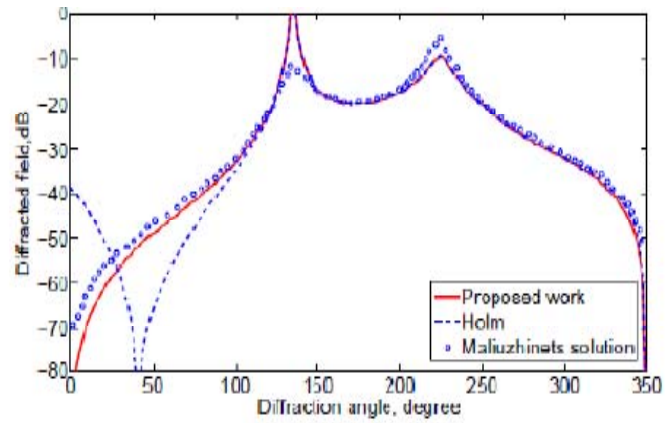

Fig. 7. Comparison of proposed diffraction coefficient with available diffraction coefficient and Rigorous Maulizhinets' solution [8]: Incident angle is $45^{\circ}$ and wedge angle is $10^{\circ}$, perpendicular polarization.

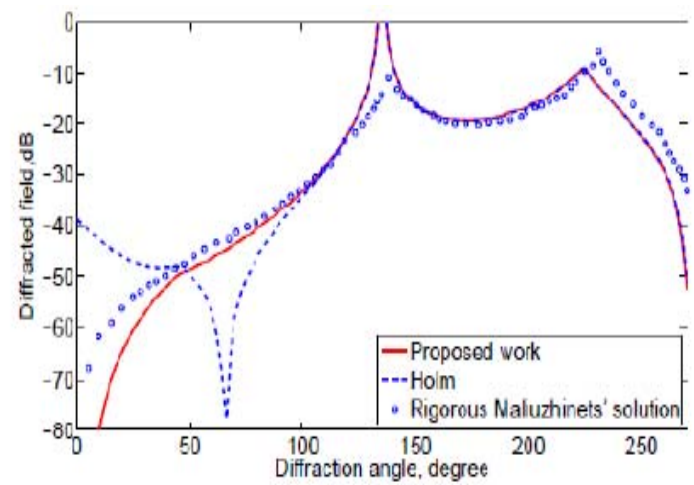

Fig. 8. Comparison of proposed diffraction coefficient with available diffraction coefficient and Rigorous Maulizhinets' solution [8]: Incident angle is $45^{\circ}$ and wedge angle is $90^{\circ}$, parallel polarization.

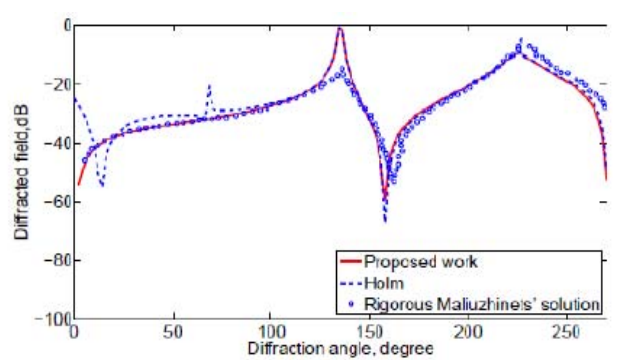

Fig. 9. Comparison of proposed diffraction coefficient with available diffraction coefficient and Rigorous Maulizhinets' solution [8]: Incident angle is $45^{\circ}$ and wedge angle is $90^{\circ}$, perpendicular polarization.

\section{Application of Proposed Diffraction Coefficient in} Arbitrary Complex Urban Scenario.

In this section, we will validate the applicability of proposed diffraction coefficient in a complex urban scenario of Ottawa city. in our analysis, we took microcellular environment (Ottawa City) for which measurement was carried out by James H. Whitteker [10]. In particular region (core) of this city, there are 15 buildings and total 72 walls (See Fig.10). This urban scenario is presented in 2D view in [[10], Fig. 2].

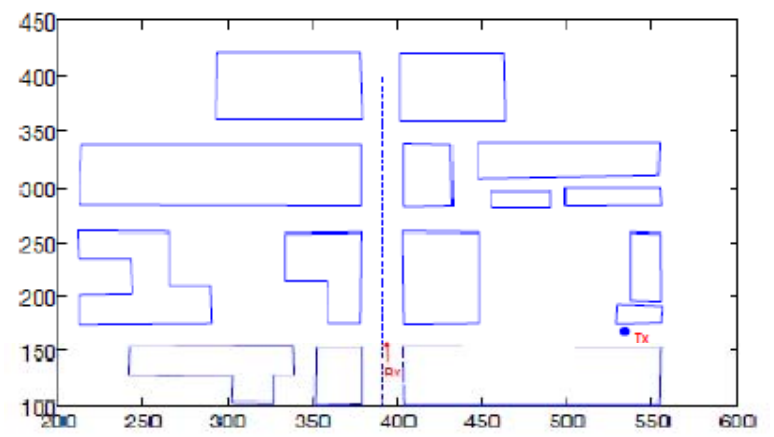

Fig. 10. Map of Ottawa city.

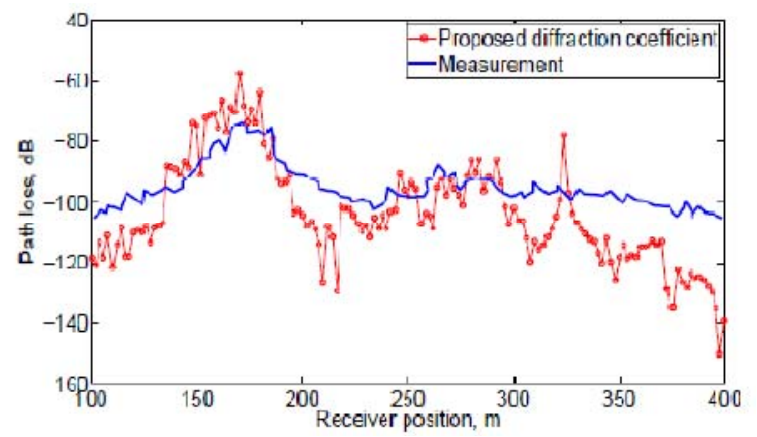

Fig. 11. Comparison of path loss prediction obtained by proposed diffraction coefficient with available measurement [10].

The measurement was carried out along the Bank St. and $\mathrm{Tx}$ is at 263 Laurier St. Frequency of operation is $910 \mathrm{MHz}$. The conductivity and permittivity of wall material were chosen to be $\sigma=0: 001 \mathrm{~S} / \mathrm{m}$ and $\mathcal{E}_{r}=7$. The value of $\mathcal{E}_{r}$ is consistent with the range $5 \leq \varepsilon_{r} \leq 7$ by direct measurement [11] and the value of $\sigma$ is consistent with [12]. To obtain the path loss prediction, image-based ray tracing engine was set to 3 reflections and 2 diffractions. The prediction result is shown in Fig. 11. The result shows the usefulness of the proposed diffraction coefficient to handle arbitrary complex scenario. 


\section{CONCLUSION}

In this paper, we have presented a novel heuristic diffraction coefficient which is perfectly symmetrical and reciprocal. The proposed coefficient is validated by comparing it with rigorous Maliuzhinets solution, Holm's heuristic diffraction coefficient. Both the parallel and perpendicular polarizations have been considered. It is noted that the proposed coefficient is more accurate in illumination region and agrees well with Holm' coefficient and Maliuzhinets solution in shadow region. Further, the coefficient has been applied in the complex urban scenario of the Ottawa city and its usefulness has been demonstrated to handle such a complex scenario.

\section{REFERENCES}

[1] J. B. Keller, "Geometrical theory of Diffraction," J. Opt. Soc. Amer. vol. 52, no. 2, pp. 116-130, Feb 1962

[2] R. G. Kouyoumjian and P. H. Pathak, "A uniform geometrical theory of diffraction for an edge in a perfectly conducting surface," in Proc. IEEE, vol. 62, no. 11, pp. 1448-1461, Nov. 1974.

[3] R. J. Luebbers, "Finite conductivity uniform UTD versus knife diffraction prediction of propagation path loss," IEEE Trans. Antennas Propagat, vol. AP-32, pp. 70-76, Jan. 1984.

[4] Kate A. Remley, Harry R. Anderson, and Andreas Weisshar, "Improving the Accuracy of Ray-Tracing Techniques for Indoor Propagation Modeling," IEEE Transactions on Vehicular Technology, Vol. 49, No. 6, Nov.2000.

[5] P. Holm, "A new heuristic UTD diffraction coefficient for nonperfectly conducting wedges," IEEE Trans. Antennas Propagat. vol. 48, no. 8, pp. 1211-1219, Aug. 2000.

[6] H. M. El-Sallabi and P. Vainikainen, "Improvement to diffraction coefficient for non-perfectly conducting wedges," IEEE Trans. Antennas Propagat, vol. 53, no. 9, pp. 1211-1219, pp. 3105-3109, September 2005.

[7] D. N. Schettino, F. J. S. Moreira, K. L. Boges, and C. G. Rego, "Novel heuristic UTD coefficients for the characterization of radio channels," IEEE Trans. Magnetics, vol 43, no.4, pp. 1301-1304, April 2007.

[8] H. M. El-Sallabi, Ioannis T. Rekanos, and P. Vainikainen, "New heuristic diffraction coefficient for lossy dielectric wedges at normal incidence," IEEE Antennas Propagat. letter, vol. 1, pp. 165-168, 2002.
[9] M. Adi and J. Lavergnat, "Comparison of Luebbers' and Maliuzhinets' wedge diffraction coefficients in urban channel modeling," Progress Electromagn. Res., pp. 1-28, PIER 33, 2001.

[10] H. James Whitteker, "Measurements of path loss at $910 \mathrm{MHz}$ for proposed microcell urban mobile systems," IEEE Trans. Veh. Technol., vol. 37, no. 3, pp. 125-129, August 1988 .

[11] O. Landdron, M. J. Feuerstein, and T. S Rappaport, "A comparison of theoretical and empirical reflection coefficients for typical exterior wall surfaces in mobile radio environment," IEEE Trans. Antennas Propagat. vol. 44, pp. 341-351, Mar. 1996.

[12] E. G. Athanasiadou and R. A. Nix, "Investigation into the sensitivity of the power predictions of a microcellular ray tracing propagation model," IEEE Trans. on Veh. Technol., vol. 49, no. 4, pp. 1140-1151, July 2000 .

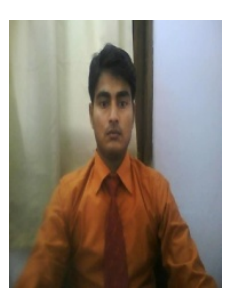

Puspraj Singh Chauhan was born in Kalpi, India. $\mathrm{He}$ received his B.Tech degree in Electronics and Communication Engineering from U.P. Technical University, Lucknow in 2009 and M.Tech degree in Digital Signal Processing from Uttarakhand Technical University, Dehradun in 2011. He is presently working as an Assistant Professor in College Of Engineering Roorkee, Uttarakhand Technical University. He is reviewer of many international journals. His main research interests are in the area of wireless channel modeling and focused on the electromagnetic wave propagation techniques

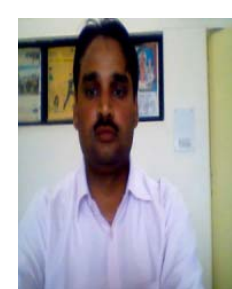

Sanjay Soni was born in UP, India on March, 1975. $\mathrm{He}$ received his Bachelor of Engineering in electronics engineering from Madan Mohan Malviya Engineering College, Gorakhpur in 1997, the M.Tech degree in communication engineering from IIT Kanpur in 2004, and $\mathrm{PhD}$ in wireless Communication Engineering from IIT Kharagpur in 2011. From 1998 to 2007 he was Assistant Professor in the department of Electronics and Communication engineering, G. B. Pant Engineering college, Pauri. From 2007 he joined IIT kharagpur as a Research Scholar to pursue his PhD. In 2010 he joined back to the same institute at the post of Associate Professor. Currently he is involved in teaching and research in the area of wireless communication. His research interest includes propagation modeling and characterization of wireless channel, time-domain analysis of propagation channel. 